\title{
Aspectos sociotécnicos da computação: contextualizando o desenvolvimento de sistemas de computação com o modelo Mikropolis
}

\author{
João Porto de Albuquerque*
}

\begin{abstract}
Resumo: O presente artigo discute a necessidade da consideração de aspectos sociotécnicos na prática científica e profissional em computação. Para tanto, uma breve retrospectiva histórica e o panorama atual da pesquisa de aspectos sociotécnicos dentro da área de computação são apresentados. Posteriormente, discute-se um exemplo de pesquisas nessa área: o modelo Mikropolis, desenvolvido com a intenção de oferecer orientação para a prática das atividades do profissional de computação em relação aos aspectos sociais dessas atividades. Além disso, são levantadas questões sobre como aproximar esse modelo da realidade no Brasil, com o intuito de obter instrumentos analíticos e didáticos particularmente apropriados ao contexto brasileiro.
\end{abstract}

\begin{abstract}
This paper discusses the need of considering sociotechnical aspects in the scientific and professional practice of computing. In the pursuit of this goal, the paper firstly places the research on sociotechnical aspects of computing in a historical context and outlines the current research scenario in the area. Subsequently, the Mikropolis Model is explained - a model developed to provide orientation to the practical activities of the computing professional in respect to the social aspects of these activities. Furthermore, the paper discusses how to bring the model closer to the particular Brazilian context, in order to achieve analytical and didactic instruments especially suited to the reality in Brazil.
\end{abstract}

\section{Introdução}

Nos tempos atuais os computadores estão cada vez mais presentes em diversos setores da atividade humana: da pesquisa científica de ponta, em praticamente todas as áreas do conhecimento, até a mais corriqueira das ações cotidianas. Esse caráter crescentemente ubíquo do artefato tecnológico computador confere, assim, às pessoas que têm na computação sua atividade profissional um papel crucial para o futuro da sociedade. Para que possa desempenhar suas atividades de acordo com essa responsabilidade, necessita o profissional de

* Department Informatics, Universität Hamburg, Vogt-Koelln-Str. 30, 22607 Hamburg

portolinformatik.uni-hamburg.de

Uma versão preliminar deste artigo foi apresentada sob o título "Por uma perspectiva sociotécnica do desenvolvimento de sistemas de computação: o exemplo do modelo Mikropolis" em [1].

A pesquisa para confecção do presente artigo foi parcialmente financiada pela Fundação Alexander von Humboldt. 
Aspectos sociotécnicos da computação: contextualizando o desenvolvimento de sistemas de computação com o modelo Mikropolis

computação não apenas possuir conhecimentos técnicos em sua área, mas também ser capaz de orientar-se com respeito aos aspectos sociais de sua atividade profissional, ou seja, deve ser consciente das inter-relações entre a atividade de computação, os artefatos tecnológicos por ela produzidos e seu mais amplo contexto social.

Essas inter-relações devem, portanto, ser pesquisadas e analisadas dentro da comunidade científica de computação, de maneira a produzir meios para que o profissional adquira essa orientação frente aos aspectos sociotécnicos de sua atividade. Entretanto, o número de pesquisas que se dedicam à analise sistemática dos aspectos sociotécnicos da atividade de computação é, na comunidade acadêmica de computação brasileira, ainda reduzido. Por conseguinte, a formação dos profissionais de computação, embora de excelência técnica, possui muitas vezes uma lacuna. $O$ presente artigo pretende contribuir na direção de preencher essa lacuna, apresentando instrumentos para a consideração dos aspectos sociotécnicos relativos ao desenvolvimento de sistemas de computação — compreendendo, aqui, tanto as atividades de projetar e desenvolver software como a de arquitetar novos meios de se utilizar computadores [2]. A base para esse empreendimento será dada pelo Modelo Mikropolis (MM), desenvolvido na Universidade de Hamburgo, o qual oferece um meio para a análise das interrelações entre a atividade de computação, os seres humanos e a sociedade global dentro de um mesmo contexto sistêmico [3, 4]. Além disso, serão levantadas questões sobre como aproximar esse modelo da realidade acadêmica e social no Brasil, com o objetivo de desenvolver instrumentos analíticos e didáticos especialmente apropriados para o contexto brasileiro.

Visando uma melhor contextualização, a próxima seção apresenta uma breve retrospectiva histórica, para que posteriormente se analise o panorama atual em pesquisas sobre os aspectos sociotécnicos das atividades de computação em nível internacional (Seção 2.1) e nacional (Seção 2.2). Na Seção 3, apresenta-se o Modelo Mikropolis (MM) e suas diferentes perspectivas. Na seqüência, a Seção 4 apresenta uma discussão do modelo, enquanto que a Seção 5 tece considerações finais sobre relação do MM com a sociedade brasileira.

\section{Aspectos sociotécnicos e a computação como disciplina}

\section{Ou: Por que (não) deveria um profissional de computação ocupar-se com os aspectos sociotécnicos de sua atividade?}

Apesar de enraizar-se em conhecimentos acumulados durante uma trajetória longa na história da ciência, a ciência da computação surge como disciplina científica e acadêmica apenas na segunda metade do século vinte. Seu surgimento nos anos sessenta nos Estados Unidos da América e em países europeus está ligado à necessidade de prover qualificação especializada em processamento de dados para pessoas que deveriam lidar com um novo artefato tecnológico: os computadores. Os primeiros cursos relacionados aos computadores 
Aspectos sociotécnicos da computação: contextualizando o desenvolvimento de sistemas de computação com o modelo Mikropolis

foram desenvolvidos por volta de 1960 nos EUA, e subdividiam-se em três linhas: computer engineering (engenharia de computadores), computer science (ciência de computadores ${ }^{1}$ ) e information science (ciência da informação) [5].

Na Europa, por outro lado, a maioria dos países seguiu a denominação francesa para a nova disciplina acadêmica: informatique ${ }^{2}$ (oriunda das palavras information e automatique ou éléctronique), definida oficialmente pela Academia Francesa em 1967 como: "ciência do tratamento racional, notadamente por máquinas automáticas, da informação considerada como suporte de conhecimento humano e de comunicação dentro dos domínios técnico, econômico e social" (Cf. [5]). Vale notar que essa definição sugere uma conotação diferente para o campo de atuação da nova disciplina, colocando o tratamento racional de informações em foco - o artefato tecnológico computador entra apenas 'notadamente' como seu meio de execução, não tendo a centralidade que o nome computer science implica —, e incluindo, ainda, fatores econômicos e sociais.

Essa diferença de nomenclatura reflete a existência de divergentes concepções e perspectivas para a nascente disciplina, as quais possuíam fronteiras e focos de atuação distintos. O que estava em jogo era, portanto, a autodefinição de uma disciplina acadêmica pela própria jovem comunidade científica atuante. Um momento exemplar nessa disputa foi a polêmica que despertou o "Relatório Denning" [7] elaborado em 1989 por uma força-tarefa conjunta da ACM (Association for Computing Machinery) e da IEEE Computer Society para definir um currículo-base para cursos do ensino superior da nova ciência. No debate - analisado em [8] e [9] —, destacam-se as posições de três renomados pesquisadores: Dijkstra, Winograd e Denning.

Dijkstra em seu clássico artigo "Sobre a crueldade de realmente ensinar ciência da computação" defende que seja erigida uma barreira de proteção entre o problema da agradabilidade (pleasantness) — isto é, a questão sobre se um mecanismo que atende a uma certa especificação é mesmo o mecanismo que gostaríamos de ter - e o problema da corretude (correctness) - isto é, a questão de como projetar um mecanismo que atenda à especificação [10]. A ciência da computação situar-se-ia, segundo Dijkstra, entre a lógica aplicada e a matemática formal, e sua tarefa consistiria em lidar com o problema da corretude, estudando a manipulação de símbolos através de programas. Para tanto, o mais importante seria provar formalmente que um certo programa cumpre sua especificação funcional (também formal). Já a questão sobre como formalizar essa especificação funcional a partir de ambientes reais — ou seja, o problema da agradabilidade — deveria ser tratada através de outros disciplinas e métodos, como por exemplo psicologia e experimentação.

\footnotetext{
${ }^{1}$ A tradução aqui é feita propositalmente de forma literal, e portanto divergente da nomenclatura brasileira "computação", com objetivo de traçar um contraste que ficará claro adiante.

${ }^{2}$ A Dinamarca constitui aqui uma exceção à regra, por ter adotado a denominação datalogia, sugerida por Peter Naur por não concordar com o excessivo acento no produto tecnológico computador da nomenclatura americana [6, p. 89].
} 
Aspectos sociotécnicos da computação: contextualizando o desenvolvimento de sistemas de computação com o modelo Mikropolis

Para Winograd o argumento de Dijkstra seria coerente e interessante, porém fundamentar-se-ia em falsas premissas: Dijkstra estaria errado sobre o que fazem computadores, sobre o que fazem programadores e sobre o que fazem engenheiros [11]. Computadores, no mundo real, seriam aparelhos utilizados para executar certas tarefas dentro de um contexto de aplicação. Para executar tais tarefas, eles de fato realizariam manipulação de símbolos, porém ela seria um meio e não um fim. Dessa forma, programadores deveriam, assim como engenheiros, possuir uma coleção de técnicas, ferramentas e projetos anteriores que os possibilitariam criar dispositivos razoavelmente confiáveis com custo e quantidade de esforços razoáveis. A ciência da computação não deveria de maneira alguma, portanto, focalizar apenas os aspectos formais da manipulação simbólica - embora esta seja uma importante ferramenta na formação do profissional de computação -, ignorando a dura escola da prática da aplicação real. Importante seria desenvolver um treinamento efetivo e rigoroso que provesse habilidades em projetar ${ }^{3}$ sistemas computacionais apropriados ao seu uso por pessoas, em analogia com o que faz a arquitetura.

Denning, o próprio coordenador daquela força-tarefa ACM/IEEE CS, retoma o debate e critica duas convicções comuns às duas posições anteriores: (a) que os requisitos para um sistema de software pudessem ser formulados de maneira inequívoca, de modo que a concordância com a especificação forneceria um critério apropriado para a avaliação do sistema; (b) que, uma vez elaborada a especificação, não seria mais necessário contato entre projetistas e usuários [12]. Essas crenças equivocadas estariam, para Denning, baseadas numa concepção taylorista de divisão do trabalho, e causariam o problema de que as especificações freqüentemente contêm requisitos ultrapassados e impedem a adaptação rápida e flexível aos requisitos em constante evolução e a situações inesperadas. Denning recomenda dar atenção às abordagens de "design participativo" e "design centrado no usuário" desenvolvidas na península escandinava.

De fato, a relação entre técnicas computacionais e a sua aplicação prática no mundo do trabalho foi particularmente cedo tema de pesquisas na Escandinávia. Já na década de 1960 houve primeiras aplicações de design participativo, em que aos empregados de uma certa companhia foi dado o direito de decidir sobre os rumos do desenvolvimento de sistemas [6, p. 120]. O norueguês Kristen Nygaard criou uma escola de pensamento cuja abordagem tornou-se internacionalmente conhecida como a abordagem escandinava (scandinavian approach). Segundo essa abordagem, o design de sistemas se inicia quando reconhecemos como falsa a idéia de que os problemas estão dados, bastando que procuremos a resposta correta para eles - idéia subjacente à visão formalista de Dijkstra. Na verdade, o projetista de

\footnotetext{
${ }^{3}$ Utilizo nesse texto, dependendo do contexto, os verbos projetar e desenhar para traduzir os termos design (inglês) e gestalten (alemão), embora nenhuma das palavras em português cubra inteiramente o campo semântico destas últimas. Assim, o leitor deve entender "projetar" e "desenhar" aqui compreendendo não só o planejamento técnico de um artefato, mas também o processo sociotécnico e artístico de criá-lo e "dar-lhe forma" - ambas conotações contidas nos termos estrangeiros Cf. [6, p. 119]. Em alguns poucos contextos, opto pelo anglicismo design (geralmente em sua forma substantivada), quando nenhuma das traduções me parece satisfatória.
} 
Aspectos sociotécnicos da computação: contextualizando o desenvolvimento de sistemas de computação com o modelo Mikropolis

sistemas computacionais, em sua atividade, define os problemas de uma determinada forma e escolhe uma solução razoável para eles. Essa abordagem inclui portanto, dimensões filosóficas, sociais e técnicas, diferenciando-se por possuir: a) uma perspectiva de conhecimento ampliada em relação ao racionalismo, b) métodos direcionados à interpretação, c) uma prática cooperativa e evolucionista de desenvolvimento de sistemas, e d) a participação na definição das condições sociais do contexto [6, p. 120]. A abordagem escandinava influenciou o surgimento de diversas perspectivas que vêem no design o cerne da atividade de um profissional da computação, como o próprio Winograd argumentaria posteriormente num trabalho conjunto com Flores [13].

Na Alemanha a discussão acerca das perspectivas para a disciplina informática (Informatik, tradução da denominação francesa) correu inicialmente sem vínculo direto com a discussão americana (embora se possam ver similaridades entre as duas), porém em diálogo com a abordagem escandinava. Autores alemães iniciaram por criticar a visão tradicional da informática que via a disciplina como sendo ancorada unicamente em suas raízes na matemática e engenharia. $\mathrm{O}$ nome escolhido para a disciplina — informática — já indicaria que essa ciência não poderia ocupar-se nem unicamente do artefato tecnológico computador, nem exclusivamente dos dados; ela deveria, na verdade, analisar os processos de formalização e automatização de processos sociais, como, por exemplo, os processos de trabalho. Os pesquisadores alemães ${ }^{4}$ chamam a atenção para o fato de que modelos, métodos e produtos da informática são co-determinados também por desenvolvimentos históricos e culturais, requisitos econômicos e por diversos atores sociais. Por outro lado, os resultados da atividade informática influenciam de volta a realidade no contexto em que são utilizados [8, p. 11-12]. Por conseguinte, a atividade profissional da informática - seja na pesquisa ou no mercado de trabalho - deveria sempre levar em conta o contexto social de aplicação em que está inserida.

Como conseqüência dessas discussões e críticas acerca da autodefinição da jovem disciplina acadêmica denominada ciência da computação (ou informática), abriram-se novos campos de pesquisa dentro da própria disciplina. Um desses campos foi a área de engenharia de software e, em particular, a subárea denominada Interfaces Humano-Computador (ver, por exemplo, [16]). Em um outro desdobramento das discussões, abriu-se também um novo campo de pesquisa que tem seu foco não nas interfaces com o usuário final, mas em uma esfera social mais ampla. Esse campo problematiza as inter-relações entre os sistemas computacionais e seu entorno social, isto é, a atividade do profissional de computação/informática é analisada dentro de seu inseparável contexto social de aplicação. O presente artigo se insere nesse último campo de pesquisas, cujo atual panorama se apresenta a seguir.

${ }^{4}$ Nesse sentido, ver por exemplo [14] e [15]. 
Aspectos sociotécnicos da computação: contextualizando o desenvolvimento de sistemas de computação com o modelo Mikropolis

\subsection{Panorama internacional atual}

Atualmente a barreira de Dijkstra entre agradabilidade/corretude foi derrubada por iniciativas que tomam como ponto de partida o fato de que sistemas de computação e seu contexto social não podem ser separados, pois se fundem em um único sistema sociotécnico. Exemplos significativos de pesquisas nessa direção podem ser encontrados nos EUA na sucessão da linha de pesquisa inaugurada pelo pioneiro Rob Kling, denominada "Informática Social" [17], ou na área correlata "Sistemas de Informação" [18]. Embora essa última esteja nos EUA freqüentemente localizada junto às Business Schools, e portanto algumas vezes mais próximas da área de Administração e Negócios, há entretanto um constante intercâmbio com departamentos de computação. Na Europa, por outro lado, a área de Sistemas de Informação possui contornos próprios e ligeiramente diferentes: localiza-se geralmente dentro de departamentos de computação (sob denominações como "informática e sociedade" [19]) e possui forte intercâmbio com as ciências sociais na assim chamada 'corrente sociotécnica' [20].

O mais novo currículo de referência da ACM/IEEE-CS para cursos de ciência da computação, por exemplo, não hesita mais em incluir explicitamente a área "Considerações Sociais e Profissionais", com tópicos considerados de importância central (core topics) como história da computação, contexto social da computação e responsabilidades éticas e sociais, entre outros [2, p. 17]. Logo, apesar de ser um grupo relativamente pequeno em comparação com as tradicionais áreas da computação — daí advindo a atualidade da discussão sobre a barreira de Dijkstra da seção anterior — a pesquisa de aspectos sociotécnicos já conquistou um espaço significativo dentro da comunidade internacional.

Para poder realmente atingir seus objetivos, as pesquisas sobre aspectos sociotécnicos da computação devem inexoravelmente assumir um cunho interdisciplinar, dialogando também com especialistas de outras áreas - como por exemplo, das humanidades e ciências sociais - e almejando mesmo à transdisciplinaridade, isto é, a romper as barreiras entre as disciplinas tradicionais (ver [21] e [22]). O corpo de estudos de aspectos sociotécnicos dentro da ciência da computação dialoga também com as áreas interdisciplinares de Estudos das relações entre Ciência, Tecnologia e Sociedade (CTS) e de Avaliação da Tecnologia (Technology Assessment).

Dentro desse diálogo, o especialista em computação pode contribuir com seu expertise para elucidar questões relativas à produção e funcionamento dos sistemas computacionais (ou, de forma mais geral, das Tecnologias de Informação e Comunicação - TICs), desfazendo mitos e incompreensões derivadas da falta de conhecimento técnico. Por outro lado, o diálogo interdisciplinar pode trazer para o profissional de computação maior consciência das dimensões éticas e sociais das tecnologias que desenvolve, possibilitando-o enxergar que o desenvolvimento tecnológico está inextrincavelmente associado à sociedade, e permitindo-o, assim, incorporar parâmetros sociais em sua prática. Do ponto de vista científico, Coy [23] aponta, ainda, que a área Informática e Sociedade pode colaborar para a análise crítica dos 
Aspectos sociotécnicos da computação: contextualizando o desenvolvimento de sistemas de computação com o modelo Mikropolis

fundamentos teóricos e da evolução da jovem ciência da computação; pois, como disse o filósofo Michel Foucault em uma de suas conferências, a diferença entre uma ciência e uma pseudo-ciência é que a primeira registra a sua própria história (cf. [5]).

\subsection{Cenário brasileiro}

No Brasil, há dentro da comunidade científica da ciência da computação ${ }^{5}$ a consciência de que o profissional de computação necessita ter "uma visão humanística crítica e consistente sobre o impacto ${ }^{6}$ de sua atuação profissional na sociedade", como prescreve o próprio currículo de referência da Sociedade Brasileira de Computação [25, p. 3]. Esse documento sugere mesmo a incorporação de uma disciplina dedicada aos aspectos sociais da computação na grade curricular [25, p. 12].

No entanto, a quantidade de pesquisadores dentro da computação que se dedica rigorosamente a esses estudos ainda é reduzida. Fato que comprova essa baixa quantidade de pesquisas na área é a escassez de publicações e material didático sobre o assunto que sejam gerados e direcionados à comunidade de ciência da computação ${ }^{7}$. Não obstante, existem primeiras iniciativas de grupos de pesquisa, bem como pesquisadores individuais dentro da comunidade brasileira de computação a se enveredar adentro do campo sociotécnico (e.g. [1,27]), como nas nascentes comunidades de Sistemas Colaborativos (vinculados à comunidade internacional Computer Supported Cooperative Work) e Sistemas de Informação.

O presente artigo vem se somar a essas notáveis iniciativas, procurando estabelecer no Brasil um campo de pesquisa que analise rigorosamente as relações entre a sociedade (especialmente a brasileira) e a computação - entendida aqui compreendendo tanto a atividade profissional e científica, como a função de computar embutida em artefatos tecnológicos. Para tanto, a próxima seção descreve o ponto de partida para a análise: a linha de pesquisa alemã, particularmente através do Modelo Mikropolis — desenvolvido na Universidade de Hamburgo por um grupo interdisciplinar de pesquisa.

\footnotetext{
${ }^{5} \mathrm{O}$ relatório Denning sugere a denominação science of computing para a nova disciplina, termo que não pegou nos EUA, mas que foi posteriormente traduzido e adotado oficialmente pela comunidade brasileira (embora em algumas universidades o nome informática seja ainda utilizado).

${ }^{6}$ Como argumenta Pannabecker[24] com base em estudos construtivistas em CTS, o uso do termo "impacto" é, na verdade, inadequado para descrever as relações entre tecnologia e a sociedade, visto que estas são muito mais complexas do que a simples relação determinística implícita na metáfora impacto.

${ }^{7}$ Estou usando aqui a mesma diferenciação feita por Kling entre cursos (e pesquisas) analíticos sérios sobre aspectos sociais da computação e outros cursos, muito mais numerosos, que dedicam uma semana ou duas para examinar algum aspecto social específico [26].
} 
Aspectos sociotécnicos da computação: contextualizando o desenvolvimento de sistemas de computação com o modelo Mikropolis

\section{O Modelo Mikropolis (MM)}

O MM é um modelo das inter-relações entre construção técnica e mudanças sociais com especial foco sobre as inovações em tecnologias de informação e comunicação. Seu objetivo principal é complementar o conhecimento técnico e especializado de profissionais e estudantes de computação com um conhecimento orientacional que os auxilie em sua prática profissional e científica [3, 4, 28]. O termo Mikropolis origina-se da fusão das palavras Mikroelektronik (microeletrônica) e polis — palavra grega que denominava as antigas cidades-estado como comunidades de cidadãos - e constitui, assim, uma metáfora para a imbricação entre tecnologia e sociedade nos dias atuais.

O MM estrutura as inter-relações computação/sociedade através de três elementos principais: a) uma perspectiva sociotécnica do design de sistemas computacionais; b) a diferenciação entre o micro e o macro-contexto; c) a descrição exemplar de opções de design passadas, presentes e futuras, como também os caminhos de utilização da técnica que foram escolhidos e aqueles que ainda estão por escolher. A seguir esses três elementos são respectivamente descritos, tomando por base [4] e [3].

\subsection{A perspectiva sociotécnica}

A perspectiva sociotécnica utilizada no MM compreende o processo de transformação de padrões de ação social para o formato de informação técnica, a qual entra novamente no espaço social, modificando-o. A primeira etapa desse processo exige uma descontextualização do padrão de ação social estabelecido, para o qual só assim se pode construir uma versão formal, por exemplo, na forma de um artefato tecnológico software, ou sistema computacional. Esse artefato será então introduzido no contexto social num processo de recontextualização, modificando-o como resultado do próprio uso da tecnologia produzida.

Examinado de forma detalhada esse processo, podem-se distinguir nele três etapas: i) formalização; ii) algoritmização; iii) utilização (ou apropriação). A formalização tem como pré-requisito a descontextualização de um padrão de ação social e vem acompanhada de um processo de destruição: a descrição de ações por meio de operações (Siefkes apud [4]). Enquanto uma ação se desenvolve em uma situação e contexto social específicos, uma operação descreve uma maneira de agir que se repete e se tornou rotineira. Essa descrição se baseia no ponto de vista do sujeito que a realiza (o observador), o qual está sempre associado a uma intenção social. No entanto, com a formalização de uma ação em uma operação — de modo que esta venha a ser posteriormente transformada em artefato tecnológico - o objetivo e contexto sociais originais da ação tornam-se invisíveis na estrutura formal resultante, embora esta os carregue em si de certa forma.

À formalização sucede a algoritmização, processo no qual ações são traduzidas em 
Aspectos sociotécnicos da computação: contextualizando o desenvolvimento de sistemas de computação com o modelo Mikropolis

um procedimento computacional, que será finalmente implementado por um programa. Esse programa resultante representa, assim, a tentativa de realização daqueles objetivos e intenções sociais que estruturaram a observação inicial, e se assenta, portanto, sobre um fundamento social. Socialmente fundamentados são, sobretudo, as motivações centrais de automatização e racionalização, as quais influenciam a determinação dos padrões de ação que serão selecionados para serem formalizados. Exemplos notáveis de motivações constituem aquelas oriundas de contextos de produção e trabalho, mas também as motivações provenientes das relações formalmente regulamentadas da vida pessoal.

Com a introdução dos programas - já desprendidos de seu contexto e sentido social originais - em processos sociais concretos, entra em cena uma imprevisível prática de utilização e apropriação que é também freqüentemente conflituosa. Usuários não acionam automaticamente as funcionalidades oferecidas pelo software, mas, pelo contrário, têm de reorganizar as suas ações tradicionais em um novo arranjo, o qual tanto pode seguir as intenções inscritas pelos desenvolvedores na tecnologia de informação disponibilizada, como também se pode guiar por motivações e interesses próprios e até certo ponto arbitrários, produzindo novos e imprevistos usos para o artefato tecnológico.

\subsection{O micro-contexto}

O micro-contexto possui dois elementos principais: o Sistema de Informática (SI) e as Organizações Usuárias de Tecnologia da Informação (TI). Por Sistema de Informática entende-se, no MM, o conjunto de atores que desenvolvem e projetam tecnologias de informação, quer pertençam esses atores a empresas produtoras de TI, quer façam parte de instituições de pesquisa e ensino. As Organizações Usuárias de TI, por sua vez, englobam empresas privadas e públicas que se utilizam dos artefatos tecnológicos gerados pelo SI. A Fig. 1 oferece uma representação gráfica das inter-relações entre esses dois elementos.

A divisão entre SI e Organizações Usuárias é feita com o intuito de auxiliar a análise, embora, na realidade, muitas vezes as fronteiras entre esses dois sistemas não sejam tão nítidas - como, por exemplo, no caso de ter uma empresa um departamento próprio de pesquisa e desenvolvimento. Tal divisão permite, entretanto, melhor descrever as inter-relações entre, de um lado, os requisitos advindos da utilização de TI, e, de outro, as ofertas provenientes do desenvolvimento tecnológico. Dessa forma, o micro-contexto do MM oferece uma perspectiva do processo de desenvolvimento, introdução e utilização de software que tem seu foco nos diferentes interesses dos atores envolvidos, nas contradições entre expectativas e a realidade, bem como em mal-entendidos recíprocos.

Analisando a parte central do modelo na Fig. 1, isto é, a dinâmica das inter-relações sociotécnicas entre SI e Organizações Usuárias, torna-se claro como essa dinâmica é importante para a produção de inovações tecnológicas. Cada um dos lados disponibiliza diversas opções 
Aspectos sociotécnicos da computação: contextualizando o desenvolvimento de sistemas de computação com o modelo Mikropolis

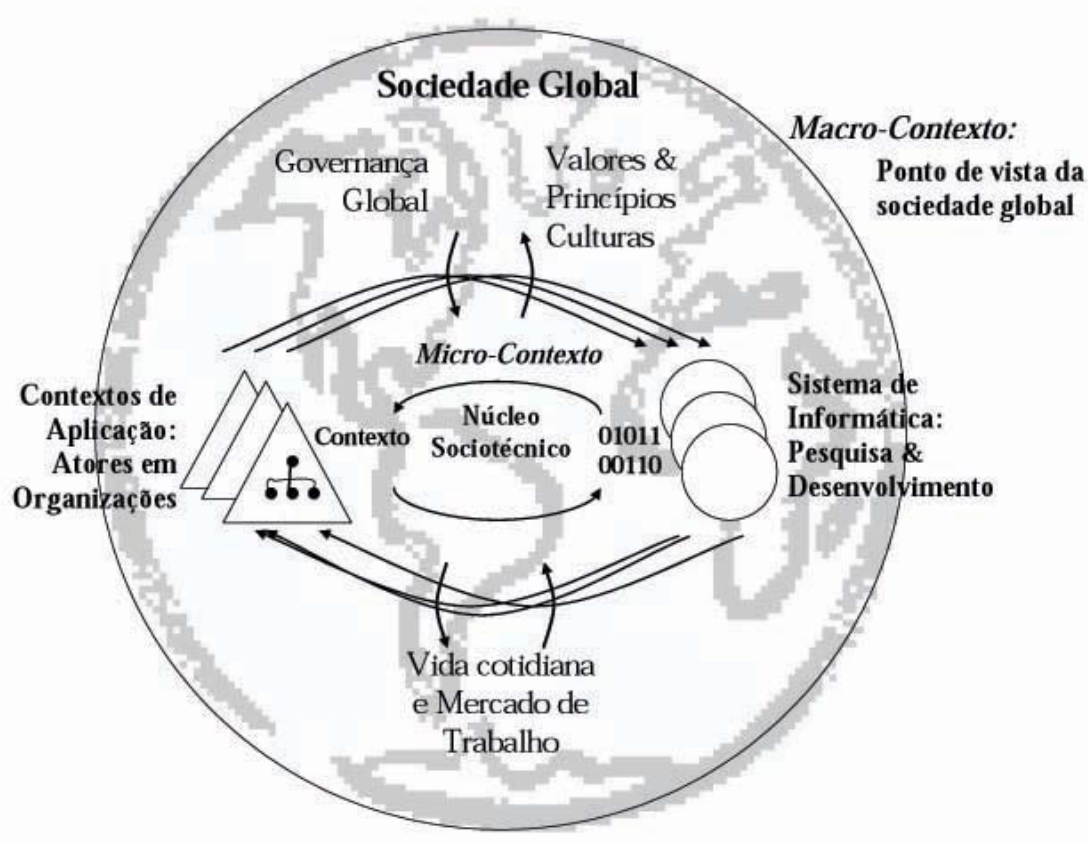

Figura 1. O Modelo Mikropolis (adaptado de [4])

de ação para o outro lado, influenciando-o. Nesse processo, o SI realiza o assim denominado "technology push": as tecnologias que são por ele desenvolvidas são divulgadas e oferecidas (por exemplo, por meio de revistas especializadas, feiras tecnológicas, etc.) às organizações, as quais procuram selecionar aquelas opções que melhor atendem aos seus requisitos. Por outro lado, as organizações também atuam ativamente no chamado "demand pull", isto é, elas enviam diversos sinais em forma de sondagens e requerimentos para produtores, desenvolvedores e cientistas do SI que, por sua vez, procuram reconhecer estratégias por detrás das demandas, buscando nichos com potenciais de sucesso para pesquisa e desenvolvimento. Da combinação desses dois movimentos surgem espirais de inovação que enlaçam os dois sistemas num movimento de expansão contínuo.

Além desse tipo de inovação, há também aquelas que são impulsionadas por outras motrizes. Desenvolvimentos posteriores do MM incluem, por exemplo, inovações geradas pela comunidade de software livre, e também aquelas que acontecem na fase de utilização, 
Aspectos sociotécnicos da computação: contextualizando o desenvolvimento de sistemas de computação com o modelo Mikropolis

quando usuários descobrem novos requisitos e formas de uso não previstos pelos produtores do software (denominadas na literatura de "innofusion" ou "learning by struggling") - as quais não serão aqui abordadas devido a restrições de espaço. O leitor interessado pode obter mais detalhes em [4].

\subsection{O macro-contexto}

No macro-contexto do MM são analisadas as inter-relações entre os desenvolvimentos sociais de maneira geral e a dinâmica de desenvolvimento tecnológico. Esse nível compreende, assim, a análise de modelos ou princípios-guia existentes na sociedade (Leitbilder), bem como valores, normas e regulamentações sociais. De fato, os elementos do microcontexto - Organizações e Sistema de Informática - encontram-se incrustados em um ambiente social maior que possui valores, tradições e sistemas científico-educacionais de características específicas em termos culturais, econômicos e jurídicos. Processos de produção e inovação tecnológica vêm, por conseguinte, freqüentemente acompanhados de tensões e adaptações sociais.

Um dos mais proeminentes exemplos dessas influências é a globalização econômica. Como argumenta Castells, embora não sejam as tecnologias de informação e comunicação (TIC) a causa do processo de transformação social em direção à globalização econômica, as TIC são, de fato, o meio viabilizador central dessas mesmas transformações [29]. Por um lado, as grandes organizações multinacionais vêem nas TIC um enorme potencial para aumentar sua competitividade num mercado global através da redução de custos e tempos de transação e da ampliação de seus espaços de ação, utilizando as tecnologias para controlar e gerenciar unidades de produção a distância. Tal fato direciona e canaliza as espirais de inovação tecnológica do micro-contexto (ver seção anterior). Por outro lado, a própria estrutura em rede - ou seja, a soma de ligações flexíveis entre nós, indivíduos, grupos, empresas ou regiões - tornou-se o novo paradigma econômico e social [29], com as TIC atuando no sentido de diminuir as distâncias temporais e espaciais entre os diversos componentes.

Dessa maneira, através do macro-contexto no MM pode-se descrever os fatores e as condições sociais específicos que são relevantes para as ações de atores nas organizações e no sistema de informática, ou seja, para o micro-contexto. Além disso, as estruturas que se desenvolvem no micro-contexto podem ser observadas na perspectiva de seu significado no sentido de trazer à tona transformações na camada social como um todo.

\subsection{Processos e Caminhos}

O último elemento do $\mathrm{MM}$ adiciona aos anteriores uma dimensão temporal, através da introdução de uma análise histórica dos desenvolvimentos tecnológicos. Os caminhos de uso da tecnologia (Techniknutzungspfad) espelham paradigmas, ideais, modelos, métodos, 
Aspectos sociotécnicos da computação: contextualizando o desenvolvimento de sistemas de computação com o modelo Mikropolis

produtos e ferramentas que se desenvolvem na sociedade, nas organizações e no sistema de informática. A metáfora caminho sinaliza, aqui, que o desenvolvimento de uma tecnologia não consiste em uma única trajetória determinada pelo progresso técnico intrínseco - como querem as visões determinista e instrumentalista da tecnologia [30, p. 48] —, mas antes em um percurso em que diversas ramificações, alternativas e opções foram e são possíveis.

No contexto do MM, a dimensão temporal está já implícita nas decisões, acontecimentos e mudanças do micro e macro contextos. A dinâmica no decorrer do tempo das interrelações entre tecnologia e fatores sociais representadas pode ser, portanto, descrita através dos caminhos de utilização. Assim, o elemento processos e caminhos descreve o desenvolvimento histórico no qual determinadas estruturas sociotécnicas foram se estabelecendo até chegar em seu estado atual, ou seja, esse último elemento do MM abrange a história das interações sociais entre os atores envolvidos ou atingidos no desenvolvimento de uma tecnologia, seus conflitos e conseqüentes perdas e ganhos de poder, seus ideais e os paradigmas técnicos vigentes. Dessa forma, a análise histórica agrega um importante valor para as perspectivas anteriores, pois o exame das condições que conduziram ao sucesso ou fracasso de certas alternativas pode oferecer lições para o desenvolvimento de novas tecnologias.

\section{Discussão do Modelo}

A premissa básica sobre a qual se assenta este artigo é a de que uma perspectiva sociotécnica do processo de desenvolvimento de sistemas computacionais, ao analisar metodicamente as inter-relações entre artefatos tecnológicos e espaço social, complementa os conteúdos tradicionais da ciência computação com o aspecto de sua inserção em práticas humanas. Essa premissa se baseia numa perspectiva da ciência da computação que a vê indo além dos domínios de suas disciplinas-mãe, a matemática e a engenharia, e abrangendo a tarefa sociotécnica do design de tecnologias que não só tenham qualidade técnica, mas que também sejam relevantes e efetivas em seu entorno social.

Dessa forma, o objetivo é contribuir para derrubar a barreira de Dijkstra (Seção 2) entre o problema da corretude - no terreno das estruturas formais e artefatos tecnológicos - e o da agradabilidade - no mais amplo universo social. Esses dois problemas estão, na realidade, inexoravelmente presentes na atividade do profissional de computação, que se movimenta em um continuum sociotécnico. Seu trabalho consiste em, como afirma Nake: "um trabalho com símbolos, os quais dentro das máquinas se transformam em sinais e que se subordinam a uma atribuição de sentido pelo ser humano. Projetar ${ }^{8}$ ambos, a atribuição de sentido e a computabilidade - aí reside a tarefa da computação como ciência: entre sinal e sentido" Nake apud [4].

${ }^{8}$ Ver nota 3 . 
Aspectos sociotécnicos da computação: contextualizando o desenvolvimento de sistemas de computação com o modelo Mikropolis

Para que esses aspectos sociotécnicos sejam efetivamente considerados durante o desenvolvimento de tecnologias, porém, é necessário integrá-los à realidade do profissional de computação. Isso significa que é necessário estabelecer um diálogo direto entre os aspectos sociotécnicos e a prática científica e profissional da computação - vale dizer, com as disciplinas clássicas da ciência da computação - , de forma que a tomada de consciência sobre a dimensão social de sua atividade realmente proporcione ao profissional de computação um conhecimento orientacional, no sentido proposto pelo filósofo Mittelstraß, isto é, a complementação do conhecimento especialista com a competência para situar suas atividades em contextos históricos, econômicos e sociais mais amplos [31]. Como afirma Rolf, essa competência pode não apenas auxiliar na identificação de caminhos técnicos relevantes para o futuro, como também oferecer segurança na avaliação de novos desenvolvimentos, aumentando mesmo a capacidade de inovação do profissional e sua responsabilidade social [32].

Além disso, ao proporcionar um olhar mais abrangente e crítico ao profissional de computação, afasta-se o risco de que uma formação exclusivamente concentrada nos aspectos técnicos gere concepções ingênuas e irrealistas sobre empresas, sociedade, homens e mulheres, tornando o especialista demasiadamente suscetível à influência de mitos e modismos, os quais muitas vezes não correspondem à realidade [32]. Recorrendo ao educador Paulo Freire, podemos dizer que o conhecimento orientacional contribui para a promoção da curiosidade ingênua - importante para despertar no educando o interesse, porém, sozinha, insuficiente - à curiosidade epistemológica — esta crítica e rigorosamente metódica [33]. Podemos encontrar, mesmo, entre as exigências do filósofo alemão Mittelstraß por um conhecimento orientacional que complemente os conhecimentos técnicos especializados uma afinidade com o pensamento de Freire, quando este afirma que: "transformar a experiência educativa em puro treinamento técnico é amesquinhar o que há de fundamentalmente humano no exercício educativo: o seu caráter formador" [33, p. 33].

O Modelo Mikropolis (MM) oferece um bom ponto de partida nesse sentido, já que a motivação de seu desenvolvimento é exatamente proporcionar um tal conhecimento orientacional. De fato, ao oferecer uma perspectiva sociotécnica da atividade da computação (Seção 3.1), integrando-a ao micro-contexto das inter-relações entre produtores e consumidores de tecnologias de informação (Seção 3.2) e, ao mesmo tempo, ao macro-contexto social (Seção 3.3) e ao caminho trilhado pelo desenvolvimento tecnológico reconstruído historicamente (Seção 3.4), o MM contribui para orientar o profissional de computação, proporcionando-lhe um instrumento analítico que o permita situar suas atividades dentro do espaço social.

A diferenciação analítica entre essas diferentes perspectivas sobre os aspectos sociotécnicos da atividade de computação é operada com o intuito de melhor elucidar aspectos diversos dessa atividade, que na prática estão sempre simultaneamente presentes formando um "tecido inconsútil" [34]. O MM possibilita, portanto, uma compreensão das relações e 
Aspectos sociotécnicos da computação: contextualizando o desenvolvimento de sistemas de computação com o modelo Mikropolis

dependências existentes entre as diversas perspectivas, isto é, ele ajuda a clarificar o quão multifacetado e retroalimentado é o processo de transformação engendrado pela interação entre tecnologias de informação, organizações e atores sociais dentro de um mundo globalizado [4].

\section{Considerações Finais}

O presente artigo apresentou argumentos para a consideração e análise dos aspectos sociotécnicos inerentes às atividades de profissionais de computação. Através de uma reconstituição histórica da definição do escopo de pesquisas na área de computação, procurou-se mostrar que houve, e há, um número significativo de vozes a defender uma visão da computação que inclua os aspectos sociotécnicos da relação entre tecnologias e práticas humanas em contextos sociais e organizacionais. Essa visão mais abrangente se justifica não apenas por um interesse prático em desenvolver tecnologias que ofereçam melhor suporte ao seu contexto, mas também pelo imperativo ético da responsabilidade do desenvolvedor pelas tecnologias que produz.

O Modelo Mikropolis (MM) aqui apresentado oferece subsídios importantes para que a comunidade brasileira de computação encare o desafio de lidar com a complexidade sociotécnica de suas atividades. Por outro lado, o MM e os instrumentos teóricos anteriormente considerados foram desenvolvidos na Alemanha em condições em certos aspectos muito distantes das necessidades particulares do Brasil e de seu contexto social próprio. Seria, portanto, interessante aproximar a análise sociotécnica do MM de conceitos oriundos de pesquisas brasileiras sobre a tecnologia, de forma a desenvolver instrumentos analíticos e didáticos especialmente apropriados para o contexto brasileiro ${ }^{9}$. Os desdobramentos dessas pesquisas poderiam ser utilizados não somente como recurso didático para uma ampla formação de profissionais de computação orientada à realidade do Brasil (em disciplinas interdisciplinares como "Computação e Sociedade" e "Sistemas de Informação em Organizações e na Sociedade"), mas também para realizar pesquisas sobre a inter-relação entre os artefatos tecnológicos (sistemas computacionais) e os aspectos sociotécnicos particulares do contexto brasileiro.

Assim fazendo, poder-se-ia contribuir para aproximar o profissional de computação no Brasil da sociedade em que se insere, dando-lhe uma consciência mais ampla acerca das dimensões sociais sempre presentes em sua atividade. Para o profissional que atua no mercado essa consciência pode aumentar sua capacidade de produzir soluções tecnológicas inovadoras que acarretem benefícios socioeconômicos, culturais e ambientais. Já para um pesquisador acadêmico, essa consciência pode mesmo colaborar para aumentar a sinergia entre o setor acadêmico e a sociedade civil (incluindo organizações públicas, empresariais e industriais)

${ }^{9}$ Nesse ponto, ver por exemplo o conceito de adequação sociotécnica (AST) [30, p. 52]. 
Aspectos sociotécnicos da computação: contextualizando o desenvolvimento de sistemas de computação com o modelo Mikropolis

- e assim, talvez, melhorar a tão criticada baixa taxa de transferência tecnológica entre esses dois setores [35, p. 129].

\section{Referências}

[1] Henrique Cukierman, editor. Anais do II Workshop Um Olhar Sociotécnico sobre a Engenharia de Software (WOSES 2006), Rio de Janeiro, 2006. PESC/COPPE/UFRJ.

[2] ACM/IEEE-CS. Association for Computing Machinery and IEEE Computer Society: The Joint Task Force for Computing Curricula 2001. Computing Curricula 2001: Computer Science, CC 2001 edition, 2001. Disponível em: http://computer.org/ curriculum em 09/02/2006.

[3] Arno Rolf. MIKROPOLIS 2010 - Menschen, Computer, Internet in der globalen Gesellschaft. Metropolis-Verlag, Marburgo, Alemanha, 2008.

[4] Detlev Krause, Arno Rolf, Marcel Christ, and Edouard Simon. Wissen, wie alles zusammenhägt: Das Mikropolis-Modell als Orietierungswerkzeug für die Gestaltung von Informationstechnik in Organisationen und Gesellschaft. Informatik Spektrum, 29(4):263-273, 2006.

[5] Wolfgang Coy. Defining discipline. In Ch. Freksa, M. Jantzen, and R. Valk, editors, Foundations of Computer Science. Springer, Berlin-Heidelberg-New York, 1997.

[6] Christiane Floyd and Herbert Klaeren. Informatik als Praxis und Wissenschaft. Tübinger Studientexte Informatik und Gesellschaft. Universität Tübingen, Tübingen, 1999.

[7] P. J. Denning, D. E. Cormer, D. E. Gries, M.C. Mulder, A. Tucker, A. J. Turner, and P. R. Young. Computing as discipline. Communications of ACM, 32(1):9-23, 1989.

[8] Arno Rolf. Das Selbstverständnis der Informatik. In Jürgen Friedrich, Thomas Herrmann, Max Peschek, and Arno Rolf, editors, Informatik und Gesellschaft, pages 8-12. Spektrum Akad. Verlag, Heildelberg; Berlin; Oxford, 1995.

[9] Wolfgang Coy and Lena Bonsiepen. Eine Curricularedebate. Informatik Spektrum, 15(6), 1992.

[10] E. W. Dijkstra. On the cruelty of really teaching computing science. In P. J. Denning, editor, Communications of ACM, volume 32, chapter A debate on teaching computing science. ACM Press, New York, NY, USA, 1989. 
Aspectos sociotécnicos da computação: contextualizando o desenvolvimento de sistemas de computação com o modelo Mikropolis

[11] Terry Winograd. Answer to dijkstra's: On the cruelty of really teaching computing science. In P. J. Denning, editor, Communications of ACM, volume 32, chapter A debate on teaching computing science. ACM Press, New York, NY, USA, 1989.

[12] P. J. Denning. Beyond formalism. American Scientist, 79(Jan.-Feb.):8-10, 1991.

[13] Terry Winograd and Fernando Flores. Understanding Computers and Cognition: A New Foundation for Design. Norwood, New Jersey, 1986.

[14] Wolfgang Coy, Frieder Nake, Jörg-Martin Pflüger, Arno Rolf, Jürgen Seetzen, Dirk Siefkes, and Reinhard Stransfeld, editors. Sichtweisen der Informatik. Theorie der Informatik. Vieweg Verlag, Braunschweig; Wiesbaden, 1992.

[15] C. Floyd, H. Züllinghoven, R. Budde, and R. Keil-Slawik, editors. Software Development and Reality Construction. Springer Verlag, Berlin, Heidelberg, 1992.

[16] Heloísa Vieira da Rocha and M. Cecília C. Baranauskas. Design e avaliação de interfaces humano-computador. Escola de Computação 2000. USP, São Paulo, 2000.

[17] Rob Kling, editor. Computerization and Controversy: Value Conflicts and Social Choices. Academic Press, San Diego, 2nd. edition, 1996.

[18] C. Avgerou. Information systems: what sort of science is it? Omega, 28:567-579, 2000.

[19] Jürgen Friedrich, Thomas Herrmann, Max Peschek, and Arno Rolf, editors. Informatik und Gesellschaft. Spektrum Akad. Verlag, Heildelberg; Berlin; Oxford, 1995.

[20] C. Avgerou, J. Siemer, and N. Bjorn-Andersen. The academic field of information systems in europe. European Journal of Information Systems, 8:136-153, 1999.

[21] Rafael Prikladnicki and Jorge Luis Audy. Construção do conhecimento e complexidade na Área de engenharia de software. In Henrique Cukierman, editor, Anais do II Workshop Um Olhar Sociotécnico sobre a Engenharia de Software (WOSES 2006), pages 51-63, Rio de Janeiro, 2006. PESC/COPPE/UFRJ.

[22] João Porto de Albuquerque and Edouard Simon. Dealing with socio-technical complexity: Towards a transdisciplinary approach to IS research. In Hubert Österle, Joachim Schelp, and Robert Winter, editors, Proceedings of the 15th European Conference on Information Systems (ECIS 2007), pages 1458-1468, St. Gallen, Switzerland, 2007.

[23] Wolfgang Coy. Was ist, was soll, was kann Informatik und Gesellschaft? In Britta Schinzel, editor, Schnittstellen: Zum Verhältnis von Informatik und Gesellschaft, Theorie der Informatik. Vieweg Verlag, Braunschweig; Wiesbaden, 1996. 
Aspectos sociotécnicos da computação: contextualizando o desenvolvimento de sistemas de computação com o modelo Mikropolis

[24] John R. Pannabecker. Technological impacts and determinism in technology education: Alternate metaphors from social constructivism. Journal of Technology Education, 3(1):43-54, 1991.

[25] SBC. Sociedade Brasileira de Computação. Currículo de Referência da Sociedade Brasileira de Computação para Cursos de Graduação em Computação e Informática versão 2003, cr99.01 edition, 2003. Disponível em: http://www.sbc.org.br/ educacao em 01/02/2006.

[26] Rob Kling. Content and pedagogy in teaching about the social aspects of computerization. In Y.J. Katz, D. Millin, and B. Offir, editors, The Impact of Information Technology: From Practice to Curriculum. Chapman \& Hall Pub Co., 1996. Disponível em: http://rkcsi.indiana.edu/archive/kling/pubs/pedagl.html em 09/02/2006.

[27] G. Rubem, J. Wainer, and T. Dwyer, editors. Informática, organizações e Sociedade no Brasil. Editora Cortez, São Paulo, 2003.

[28] E. Simon, M. Janneck, and D. Gumm. Understanding socio-technical change: Towards a multidisciplinary approach. In J. Berleur, M. I. Numinen, and J. Impagliazzo, editors, IFIP International Federation for Information Processing, Volume 223, Social Informatics: An Information Society for All? In Remembrance of Rob Kling, pages 469-479, Boston, 2006. Springer.

[29] M. Castells. A Sociedade em Rede. Editora Paz e Terra, São Paulo, 1999.

[30] Renato Dagnino, Flávio Cruvinel Brandao, and Henrique Tahan Novaes. Sobre o marco analítico-conceitual da tecnologia social. In Tecnologia social: uma estratégia para o desenvolvimento. Fundação Banco do Brasil, Rio de Janeiro, 2004.

[31] Jürgen Mittelstraß. Der unheimliche Ort der Geisteswissenschaften. In Ulrich Engler, editor, Zweites Stuttgarter Bildungsforum, Reden der Veranstaltung der Universität Stuttgart am 27. Juni 1994. Universitätsbibliothek,, Stuttgart, 1995.

[32] Arno Rolf. Orientierungskompetenz - Wege, Hindernisse, Potenziale. Managementforschung, 16:259-284, 2006.

[33] Paulo Freire. Pedagogia da Autonomia: saberes necessários à prática educativa. Paz e Terra, São Paulo, 1996.

[34] T. P. Hughes. Networks of Power: Electrification in Western Society, 1880-1930. Johns Hopkins University Press, Baltimore, 1983.

[35] Tadao Takahashi., editor. Sociedade da informação no Brasil : livro verde. Ministério da Ciência e Tecnologia,, Brasília, 2000. 